\title{
Severe neutropenia and thrombocytopenia in a young adult: a case-report
}

\author{
Clara Gomes ${ }^{1}$, Neuza Soares ${ }^{1}$, Rui Bergantim², Abílio-Vilas Boas ${ }^{1}$ \\ ${ }^{1}$ Internal Medicine Department, Centro Hospitalar Universitário de São João, Porto, Portugal \\ ${ }^{2}$ Haematology Department, Centro Hospitalar Universitário de São João, Porto, Portugal
}

\begin{abstract}
We present the case of a 20 -year-old male hospitalized with severe neutropenia and thrombocytopenia. After exclusion of other etiologies, it was concluded to be of autoimmune etiology. The prompt and sustained response of both cell lines to corticosteroids, reinforced this diagnosis. This case illustrates a very rare association of severe primary neutropenia and thrombocytopenia. The lack of serological marker makes this diagnosis delayed and resource-consuming.

Keywords: Neutropenia, thrombocytopenia, autoimmune cytopenia
\end{abstract}

\section{BACKGROUND}

Blood cell cytopenias can be primary or secondary. Primary cytopenias are more common in infants and children, being mostly a benign condition with a self-limited course ${ }^{1,2}$. In 1949 Evans and Duane ${ }^{3}$ first reported the association of acquired hemolytic anemia and other cytopenias. Platelets and erythrocytes are known to be the most affected cell lineages, with neutropenia being not so often reported ${ }^{4,5}$. Bux et al. describe $8.4 \%$ of autoimmune thrombocytopenia in a group of 143 patients with autoimmune neutropenia ${ }^{6}$. Secondary cytopenias are more common and associated with other conditions, such as autoimmune diseases, infections, drugs or hematological malignancies.

We present the case of a young adult with an upper respiratory airway infection that was treated with antibiotics before severe thrombocytopenia and neutropenia were known.

\section{CASE PRESENTATION}

A 20 year-old male, with a past history of amidgalectomy and adenoidectomy in 2003 due to recurrent upper respiratory infections, presented to his family doctor with odynophagia and fever (38.5-39. $0^{\circ} \mathrm{C}$ axillary temperature). Symptoms initially improved and fever resolved after treatment with penicillin $G$ benzathine and azithromycin, but right after he developed labial lesions resembling herpes simplex. Six days after the first medical observation he went to the emergency department because of recurrent fever and appearance of a cutaneous rash and inner lip mucosal lesions (Figure 1). At physical examination he was afebrile $\left(37.1^{\circ} \mathrm{C}\right.$ auricular temperature), hemodynamically stable (blood pressure 139/89 mmHg, heart rate $90 \mathrm{bpm}$ ), showing no signs of respiratory distress; skin and mucosal membranes were hydrated, pink, with small petechiae through the limbs and trunk; oral cavity showed two ulcerations on the inner surface of the lower lip (one of them with dark appearance) and labial herpes scar on the upper lip (Figure 1); gingival mucosa was friable; otoscopy was normal; oropharynx showed erythema but no exudates;
Figure 1. Mucosal ulceration and friable gingival mucosa.

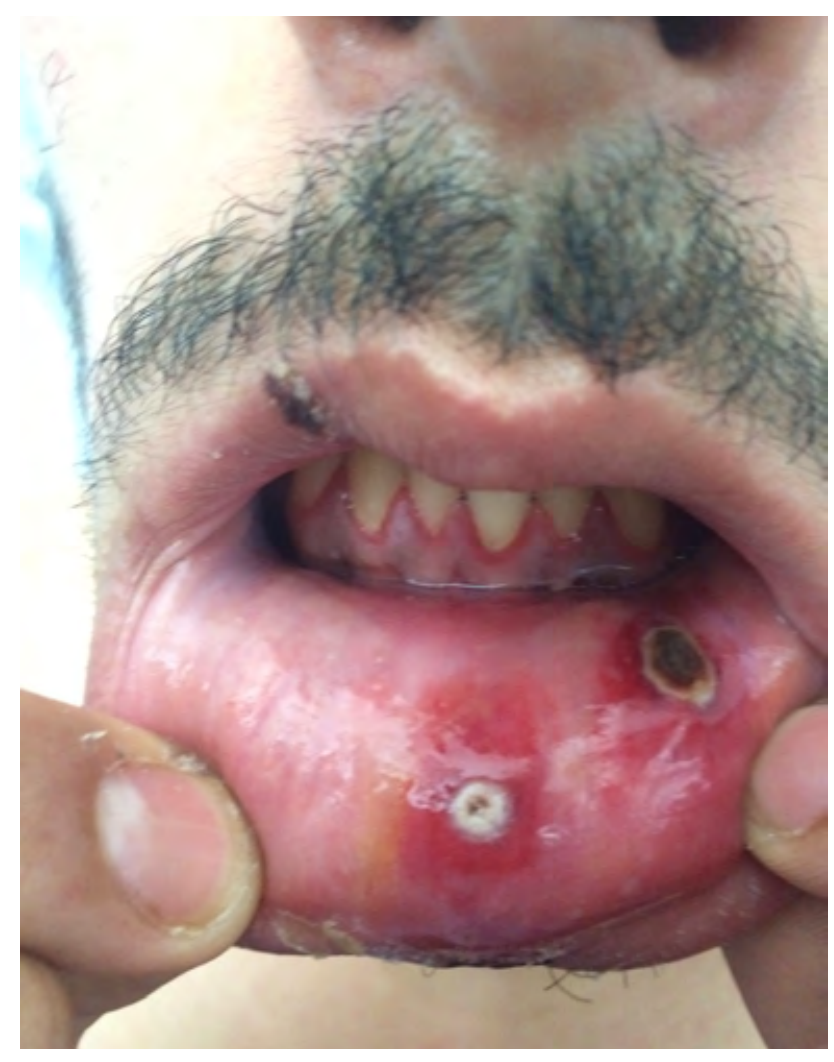

a right non-tender, soft, about $3 \mathrm{~cm}$ long axis axillary adenopathy was palpable; heart and lungs sounds were normal; abdomen was tender and painless, with no palpable masses or organomegaly; no peripheral edema.

Blood tests showed severe neutropenia $\left(0.020 \times 10^{9}\right.$ neutrophils/uL) and thrombocytopenia ( $<10 \times 10^{9}$ platelets/uL); hemoglobin level was normal (13.6 g/dL); erythrocyte sedimentation rate was $67 \mathrm{~mm} / 1^{\text {sth }}$ and $\mathrm{C}$-reactive protein was $33.7 \mathrm{mg} / \mathrm{L}$; no significant change was found on liver panel, kidney function tests, electrolytes or coagulation tests; no folate or cyanocobalamin deficit. A blood smear confirmed decreased number of leukocytes (showing reactive mono- 
cytes), decreased platelet count (with anisocytosis and megathrombocytes); no evident schizocytes or circulating blasts. Bone marrow was normocellular, with normal megakaryocytes morphology, myeloid left shift with reactive precursors, no blasts excess or maturation stop; monocytes, lymphocytes and eosinophils were morphologically normal; immunophenotyping showed no monoclonal cell populations, but a slight increase in immature granulocytic forms. Immunoglobulin levels and protein electrophoresis were normal. Human immunodeficiency virus (HIV), hepatitis B virus (HBV) and hepatitis C virus (HCV) directed antibodies and heterophile antibody test for Epstein-Barr virus (EBV) were negative. Blood cultures and the rest of microbiological study were also negative. Antinuclear antibodies (ANA) titer was 1/100 with speckled pattern and anti-thyroglobulin antibody was slightly above the normal range; no other relevant finding on autoimmune study. Chest $X$-ray was normal. Abdominal ultrasound showed homogeneous hepatosplenomegaly $(18.5 \mathrm{~cm}$ and $14.5 \mathrm{~cm}$, respectively, greatest diameter) and computerized tomography scan confirmed the slightly increased liver and spleen (13.6x15.1 x5.3 $\mathrm{cm})$. Axillary ultrassound showed a few adenopathies on the right side, the two most evident with $32 \times 17 \mathrm{~mm}$ and $35 \times 14$ $\mathrm{mm}$ diameters. Fine-needle aspiration of one of the enlarged lymph nodes found nothing but reactive changes on cytologic examination and immunophenotyping revealed polyclonal $\mathrm{B}$ cell population.

As the patient was clinically stable and no signs of critical illness were present, only supportive treatment was given while performing diagnostic workup. We expected spontaneous recovery after a presumed infectious or drug-related etiology, because of the initial presentation. However, over the time (29 days), blood cell counts showed no improvement. He had no fever or sign of active bacterial infection, besides a catheter-associated phlebitis that he developed later and was treated with flucloxacillin. He presented minor gingival bleeding and small petechiae.

Neutropenia and thrombocytopenia persisted severely decreased, as shown in Figures 2 and 3. After the first week

Figure 2. Neutrophil count during hospital stay and follow up. ${ }^{*}$ Treatment with G-CSF; • Treatment with corticosteroids.

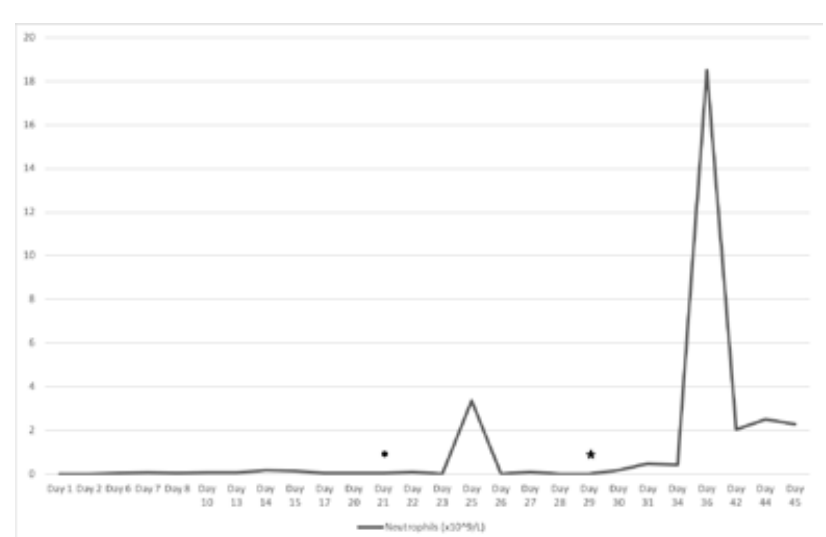

platelets seemed to be spontaneously increasing, but fell right after to values $<10 \times 10 \% / \mathrm{L}$. Platelets were transfused whenever thought clinically necessary. With granulocyte-colony stimulating factor (G-CSF), neutrophils would increase but only slightly and fall back to baseline levels (Figure 2). With lack of recovery, at day 14 , a new bone marrow aspirate was performed, as well as a bone marrow trephine biopsy. Both exams were consistent with the first observation revealing a reactive marrow and with no signs of a primary hematologic disease or infiltrative process. After 29 days, considering the lack of spontaneous recovery and no evidence of other etiology, corticosteroids were started (methylprednisolone 500 $\mathrm{mg}$ during 3 days and prednisolone $1 \mathrm{mg} / \mathrm{Kg} /$ day thereafter). A prompt and sustained increase was observed after 1 day on platelet counts, and 5 days on absolute neutrophil counts. The patient was discharged home with prednisolone $1 \mathrm{mg} /$ $\mathrm{Kg} /$ day and, after two weeks, started a slow weaning process that lasted for 6 months. After 8 months with no immunosuppressant drugs, the patient shows normal absolute neutrophil counts of $2.58 \times 10^{9} / \mathrm{L}$ and decreased but stable platelet counts of $80 \times 10^{\circ} / \mathrm{L}$. Autoimmune study was repeated and negative.

\section{DISCUSSION}

We present the case of a young patient that showed seriously reduced blood cell counts. Because of the initial upper respiratory infection and use of antibiotics, infection and drug exposure were the first diagnostic hypothesis. Both could result in bone marrow insult or induce peripheral cell destruction. Invasion of blood marrow (e.g. fungal or tuberculous) or bone marrow suppression (e.g. HIV, hepatitis, EBV) could cause decreased hematopoiesis, and EBV could also explain peripheral destruction through splenomegaly ${ }^{7,8}$. Also, mucosal ulcerations suggested a low bone marrow reserve, with inability to deliver neutrophils to the periphery and increased risk of infection, this relation being better stablished in chemotherapy-induced neutropenia ${ }^{9}$. In our patient, bone marrow showed normal cellularity and we found no infectious

Figure 3. Platelet count during hospital stay and follow up.

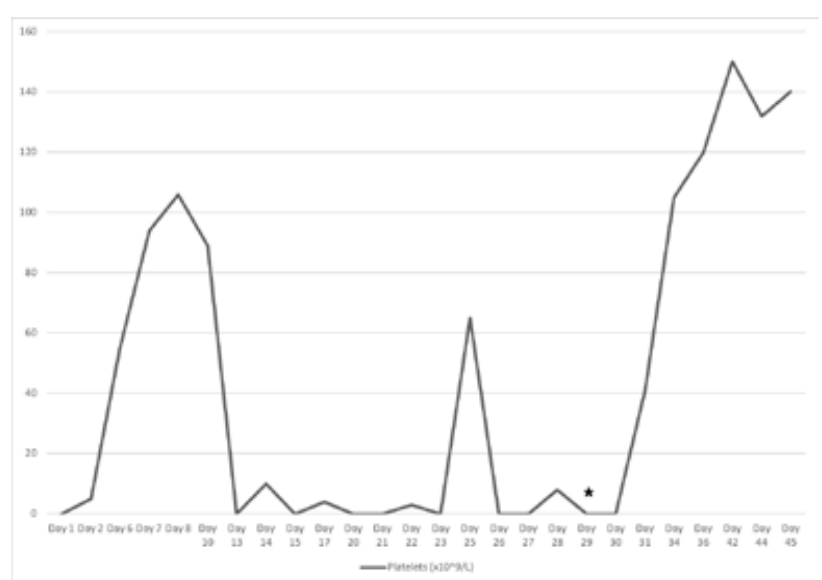


responsible agent. There are reports of agranulocytosis after treatment with azithromycin ${ }^{10}$, and it is known that penicillin can cause an immune reaction through hapten-induced antibodies, being more often associated with hemolytic anemia and, although rarely, also with thrombocytopenia and neutropenia ${ }^{11,12}$. However, we would expect some recovery after discontinuation of this drugs.

Our patient showed no symptoms suggestive of localized or systemic autoimmune disease and serological markers of autoimmunity were negative, with the exception of slight and non-specific increase in ANA and anti-thyroglobulin antibodies, which were negative during follow-up.

There was no evidence of underlying hematological malignancy: bone marrow and lymph node cytology and immunophenotyping showed no evidence of dysplasia, architectural disruption, infiltrative process or monoclonal population. We did not perform spleen biopsy, but the lack of lymphocytosis in the peripheral blood and in bone marrow, the absence M-protein or monoclonal population, as well as the clinical course, suggested that the presence of lymphoid neoplasms was unlikely. Splenomegaly would be explained by increased phagocytosis of opsonized neutrophils in the spleen or by extramedullary hematopoiesis, which is known to occur in conditions unrelated to bone marrow hematopoietic function but due to increased demand of blood cells, such as infection (like brucellosis or EBV), inflammation (as occurs in systemic lupus erythematosus or rheumatoid arthritis) or hypoxia ${ }^{13}$.

Waiting for spontaneous recovery of blood cell counts was crucial to support the autoimmune hypothesis and the need of corticosteroids for recovery, and the immediate and sustained improvement of blood cell counts after immunosuppressive therapy was key for the final diagnosis of primary immune combined cytopenia.

\section{CONCLUSION}

Autoimmune neutropenia and thrombocytopenia are a rare association, especially with the severity presented in this case. Our diagnostic workup was performed carefully in order to exclude an underlying disorder. There is no specific serological marker for this condition, and the diagnostic process may be delayed, costly and resource-consuming. Further investigation is needed. The patient should be followed in order to monitor any recurrence of important cytopenia and any other sign of systemic or local autoimmune disease.

\section{REFERENCES}

1. Farruggia $P$, Dufour $C$. Diagnosis and management of primary autoimmune neutropenia in children: Insights for clinicians. Ther Adv Hematol 6:15-24,2015.

2. Frederiksen $H$, Schmidt $K$. The incidence of idiopathic thrombocytopenic purpura in adults increases with age. Blood. 1999:94: 909-913.

3. Evans RS, Duane RT. Acquired hemolytic anemia: relation of erythrocyte antibody to activity of the disease, significance of thrombocytopenia and leukopenia. Blood 1949; 4:1196-213.

4. Pui CH, Wilimas J, Wang W. Evans syndrome in childhood. J Pediatr. 1980 Nov;97(5):754-8.

5. Mathew P, Chen G, Wang W. Evans syndrome: results of a national survey. J Pediatr Hematol Oncol. 1997 Sep-Oct;19(5):433-7.

6. Bux J, Kissel K, Nowak K, Spengel V, Mueller-Eckhardt C. Autoimmune neutropenia: clinical and laboratory studies in 143 patients. Ann Hematol 1991;63: 249-52.

7. Jain A, Naniwadekar M. An etiological reappraisal of pancytopenia - largest series reported to date from a single tertiary care teaching hospital. BMC Hematol 2013; 13:10.

8. Weinzieri EP, Arber DA. Bone marrow evaluation in new-onset pancytopenia. Hum Pathol 2013; 44:1154.

9. Blijlevens NMA, Logan RM, Netea MG.Mucositis: from febrile neutropenia to febrile mucositis. J Antimicrob Chemother 2009;63: i36-i40.

10. Kajiguchi T, Ohno T. Azithomycin-related agranulocytosis in an elderly man with acute otitis media. Inter Med 2009;48:1089-1091.

11. Aster RH, Curtis BR, McFarland JG.Drug-induced immune thrombocytopenia: pathogenesis, diagnosis and management. J Thromb Haemost. 2009 June; 7(6):911-918.

12. Neftel KA, Wälti M, Spengler $H$, von Felten $A$, Weitzman $S A$, Bürgi $H$, Weck AL. Neutropenia after penicillins: toxic or immune-mediated? Klin Wochenschr 1981;59: 877-888.

13. Kim CH. Homeostatic and pathogenic extramedullary hematopoiesis. J Blood Med. 2010;1: 13-19. 